\title{
Report of the Annual Meeting
}

Rapports annuels de la Société historique du Canada

\section{Membership of the Canadian Historical Association}

Volume 44, numéro 1, 1965

URI : https://id.erudit.org/iderudit/300647ar

DOI : https://doi.org/10.7202/300647ar

Aller au sommaire du numéro

Éditeur(s)

The Canadian Historical Association/La Société historique du Canada

ISSN

0317-0594 (imprimé)

1712-9095 (numérique)

Découvrir la revue

Citer ce document

(1965). Membership of the Canadian Historical Association. Report of the Annual Meeting / Rapports annuels de la Société historique du Canada, 44(1), 163-175. https://doi.org/10.7202/300647ar d'utilisation que vous pouvez consulter en ligne.

https://apropos.erudit.org/fr/usagers/politique-dutilisation/ 


\title{
MEMBERSHIP OF THE CANADIAN HISTORICAL ASSOCIATION
}

\author{
HONORARY LIFE MEMBERS - MEMBRES HONORAIRES $A$ VIE
}

Fee, Norman, 712 Echo Drive, Ottawa.

Groulx, M. le Chanoine Lionel, 261, avenue Bloomfield, Outremont, P.Q.

\author{
Maheux, M. l'abbé A., \\ Archiviste, Séminaire de \\ Québec.
}

\author{
Massey, Rt. Hon. Vincent, \\ Batterwood House, Port \\ Hope, Ont.
}

\section{LIFE MEMBERS - MEMBRES $\boldsymbol{A}$ VIE}

Barr, R. B. F., 43 Admiral Road, Toronto 5.

Burt, A. L., 1539 East River Terrace, Minneapolis 14, Minn.

Chartier, Mgr Emile, 605, rue Villeneuve, Sherbrooke Est, Qué.

Child, A. J. E., 402 Bate Cr., Saskatoon.

Davidson, Edgar, 32 Thurlow Road, Hampstead, Montréal.

Duffett, Miss Margaret, 506 Driveway, Ottawa.

Froats, Cecil S., 77 Gloucester St., Ottawa.

Glazebrook 'G. de T., Dept. of History, Univ. of Toronto, Toronto.

Graham. G. S., King's Col. Strand, London W.C. 2 , England.

Guillaume, Miss Sandra, 276 St. George St." Toronto 5. St. George St., Toronto 5.
Helstrom, C. T. E., Box 27. Gray, Saskatchewan.

Hudson's Bay Company, Canadian Committee Ófice, Winnipeg.

Lanctôt, G., 5642 Woodbury Ave., Montreal.

Landon, F., 128 St. James St., Apt. 308, London, Ont.
Lower, A. R. M., R.R. 3, Collins Bay, Ont.

Macdonald, Norman, 266 Market Street, Burlington, Ont.

Mackenzie, D. R., 339 Island Park Drive, Ottawa.

Mathews, Mrs. H. C., 114 King Street, Oakville, Ont. Moir, J. S., Carleton Univ. Ottawa.

Moore, Miss K. V., 157 Harris Ave., London, Ont. Morgan, C. F., Morgan Trust Co., 1455 Union Ave. Montreal.

Myers, L. P., 89 Durie St.,

Neatby, Dr. Hilda M., Univ., of Sask., Saskatoon.

Ostry, Bernard, Five Oaks, Aylmer Road, R.R. 1, Hull, P.Q.

Peake, Rev. F. A., Director Religious Education, Christ Church Rectory, Glanworth. Ont.

Raymond, Hon. D., The Senate, Ottawa.

Saunders, Dr. Richard M., Dept. of History, Univ. of Toronto, Toronto 5 .
Scott, S. Morley, c/o Dept. of External Affairs, East Block, Ottawa 2

Soward, Dean Fred H., Univ. of British Columbia, Vancouver 8.

Stacey, Coi. C.P., 874 Ave. Road, Toronto 7 .

Tombs, Guy, 1083 Beaver Hall Hill, Montreal 1.

Tombs, Laurence C., 1035 Beaver Hall Hill, Montreal 1.

Tufnell, W. G., 35 Glenridge Ave., St. Catherines, Ont.

Tweed, T. W., 34 Roff St., Toronto $2 \mathrm{~B}$

Underhill, F. H., 1.16 Springfield Road, Ottawa.

Wade, Mason, Dept. of Hade, Mason, Dept. of History, Middlesex Colyege,
Univ. of Western Ontario, London, Ont.

Wickenden, Nicholas, 11609 Saskatchewan Drive, Edmonton, Alberta.

Wilson, Dean George E. Dalhousier Univ., Halifax, N.S

Wright, Mrs. E. C.. 407 Island Park Drive, Ottawa.

Yusyk, P., 1122 Hector Bay East, Winnipeg 9

\section{ANNUAL MEMBERS - MEMBRES A L'ANNÉE}

Abraham, William P., Trinity East, Trinity Bay, Newfoundland.

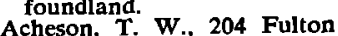

Acheson, T. W., 204 Fulton

Adams, Eric G., Suite 203, 95 King St. E., Toronto 1 . Adamson, Alan H., History Department, Sir George Williams Univ., Montreal 25.

Adey, Albert W., 63 Avenue Road, Ottawa 1.

Adsett, K. W., 1264 Stancil Lane. Victoria, B.C.

Ager, E. R. N., 2217 Webster Ave, Ottawa 8.

Akerstream, J, J. B., Dept. of History, Brandon College, Brandon, Manitoba

Akrigg, Mrs. Philip, 4633 West Eight Ave., Vancouver 8.
Alcock, Dr. F. J., 398 Third Ave., Ottawa.

Allan, F. W., 70 Dawson Road, Orangeville, Ont. Allen, Dr. A. R., Deot. of History, Univ. of SaskatHistory, Univ.

chewan, Regina. 641, Sackville. N.B.

Amtmann, Bernard, 750 Sherbrooke St. W., Montreal. Anderson, Samuel I. A., A.M., Dept. of History, A.M., Dept. St. Catharines. Ridley Col.. St. Catharines.
Anderson, Miss Susan, Box 759, Aylmer, Ont.

Anderson. Maj. Gen. W.A., 17 Westward Way, Ottawa 7.

Andreassen. John, 65, rue StLouis, St-Eustache, P.O. Archer, John H. Apt. 4, 570 Milton St., Montreal.
Ard, Ronald J.. 548 Land's End Road, R.R. 1, Sidney, B.C.

Armstrong, F. H., Dept. of History, Middlesex College, Univ. of Western Ontario, London, Ont.

Arnold, Brian J., 1339 Wilton Ave., London, Ont.

Arthur, Miss M. Elizabeth, 322 South Archibald St. Fort William, Ont.

Atherton, J., Public Archives of Canada, Ottawa. Aubrey, Roger, 411 Piux XII, Eastview, Ont.

Audet, Dr. Louis Philippe, C.P. 238, 155, rue Boulanger, Saint-Bruno de Montarville, P.Q.

Austin, Morris C., 315 Vimy St., Sherbrooke, P.Q. 
Avakumovic, Prof. Ivan, Dept. of Pol. Science, Univ. of British Columbia, Vancouver 8.

Ayre, Donald E., Registrar, Lakehead College, Port Arthur, Ont.

Babcock, R. H., R.D. 2, Altamont, N.Y.

Bailey, Alfred, Univ. of New Brunswick, Fredericton, N.B

Baker, M. K., Historic Sites Division, Dept. of Natura Resources, Government Administration Building, Regina.

Baldwin, J. R., 485 Broadview Avenue, Ottawa 13.

Ballantyne, Murray G., 355 St. James St. West, Suite 500, Montreal.

Ballard, Brook B., Jr., Principia Col., Elsah, Illinois 62028.

Banks, John, Public Archives of Canada, Ottawa.

Banks, Miss Margaret A. 377 Wharncliffe Rd. North, London, Ont.

Barrett, William G., 31 Duke St., Stratford, Ónt.

Barron, George D., 1456 Edgecliffe Ave., Ottawa 3.

Barry, Lt. W. R., Kenaston

Barry, Lt. W. R., Kenaston
Blvd., \& Blvd., \& 9 .

Baudry, Rev. René, c.s.c. 58 , rue N.-D. des Champs, Paris VIe, France.

Bear, M. Róss, 96 Stratford Cresc., Toronto 12

Beaton, A. M., Methods Analyst, 123 'Slater St. Ottawa.

Beattie, Richard, Lenark House, Carleton Univ. Ottawa.

Beatty, David P., 2092 Dietz Road, R. 3, Williamston, Michigan.

Beck, J. M., Lunenburg, N.S

Beer, Charles, Pickering Col lege, Newmarket, Ont.

Beer, D. R. Dept. of History Univ. of New England, Armidale, N.S.W., Australia.

Beggs. Don G., 705, 1st Ave. N.W., Weyburn, Sask.

Bégin, J.-O. S.J., 261 NotreDame, Sudbury, Ont.

Bélanger, Noël, prêtre. Séminaire, Rimouski, P.Q

Bell, C. A., 145 Younge St South, Aurora, Ont.

Bell, John C., 4698 Harwood Ave., Powell River, B.C.

Bell, Hon. R. A., Fairfields Farm, R.R. 2, Bell's Corners, Ont.

Belleau, Major G. de M., Le Musée Royal $22^{\circ}$ Régiment. La Citadelle, P.Q.

Benford, Allan, 113 Appel St., London, Ont.

Benson, Miss L. R., 128 St. James St.. Apt. 208 James St.,

Berelsford, Peter, 180 West Keith Road, North Vanconver, B.C.
Berger, Carl C., Dept. of History, Univ. of Toronto Toronto 5

Bernard, Jean-Paul, Collège Ste-Croix, 3800 Sherbrooke est, Montréal.

Best, Henry B. M., Moffat, Ont

Bettson, G. E., 27 Urbandale Ave., Willowdale, Ont.

Bieler, Mme J. H., 2151 Brulart, Québec 6.

Bigras, Lucien, 10587 Armand-Lavergne, MontréalNord.

Bilsland, W. W., Public Archives of Canada, Ottawa.

Black III, Prof. Robert C., Dept. of History, Trinity College, Hartford 6, Conn.

Blackley, Dr. F. D., Univ. of Alberta, Edmonton.

Blain, Prof. Jean, Dept. of History, Univ, of Montreal, Blvd. Mont-Royal, Montreal..

Blackeley, Miss Phyllis $\mathbf{R}$. Public Archives of Nova Scotia, Halifax.

Blanchard, Frances C., 6 Belmont St., Chariottetown, P.E.I.

Blaney, R. John, 67 Sargent Rd., Georgetown, Ont.

Bliss, J. M., Queen's Court, 52 Thorncliffe Park, Toron to 17

Bobr-Tylingo, Stanislaw, St. Mary's Univ., Halifax.

Bocking, D. H.. 2226 Cumberland Ave. S., Saskatoon.

Bogle, Don, 630 Huron St. Apt. 305, London, Ont.

Apt. 305, London, Ont.
Boland, Rev. F., Assumption Boland, Rev. F.. Assumption
Univ. of Windsor, 400 Huron Line, Windsor, Ont

Bolger, Rev. Francis W. P., St. Dunstan's Univ., Charlottetown, P.E.I

Boltuck, I., Quality Press Limited, 1030 St. Alexander St., Montreal.

Bonar, Jámes C., 599 Lans downe Ave., Westmount 6 , $\mathbf{P . Q}$.

Bonaventure, Brother, F. S. C., LaSalle College, Philadelphia 4, $\mathrm{Pa}$

Bond, Major C. C. J., 11 Fairhaven Way, Ottawa 2

Bosnitch, Sava D., Carleton Hall, Univ. of New Brunswick, Fredericton.

Bosson, Prof. John D., 233 Gladstone Road, 'Pitts burg, Pa. 15217.

Bouchard. Benoit. L.esL. C.P. 941, 264 boul. Marcotte, Roberval, P.Q.

Bonchard, Paul, 50, rue Aberdeen, Québec 6.

Boncher, Serge, 1 Acacia. Ottawa, Ont

Boudreau, Prof. Joseph A., Dept. of History, Univ. of Alberta, Calgary.

Boulianne, Réal G., 4847 Des Erables, Montréal.

Bourque, Miss Juliette, PubIic Archives of Canada, Ottawa.
Boutilier, Miss Helen R., 206 - 2820 Birch St., Vancouver 9.

Bovey, John A., 300 Cooper St., Ottawa 4

Bowick, Librarian, Tofield School Dist. No. 1939 Tofield, Alberta.

Bowler, E. B., 9 Beechwood Road, Wellesley, Mass.

Bowler, R. Arthur, 4A The Grove, Finchley, London N 3, England.

Bowman, J. Elgin, R.R. 4, Winchester, Ont.

Bowman, Murray, No. 301 , 1065 West $12^{\text {th }}$, Vancouver

Bowsfield, Hartwell, Provincial Archivist, Provincial Library, Winnipeg 1.

Boyce, Geral E., 173 Bridge St East, Belleville, Ont.

Boylan, D. B., Legislative Librarian, Box 848, Charlottetown, P.E.I.

Brady, Prof. Alex., Dept. of Political Economy, Univ. of Toronto, Toronto 5

Bragdon. Chandler, 11 Pleasant St., Plattsburg, N.Y

Braginetz, Miss Elizabeth, 3954 Maplewond Ave., Montreal.

Brain, Miss Elizabeth, Public Archives of Canada, Ottawa.

Bault, Dr. Lucien, 27, Boucherville, Hull, P.Q.

Brehaut, Mrs. W. M., 7 Crafton St., Charlottetown P.E.I.

Brehaut, Willard, Ontario Inst. for Studies in' Education, 344 Bloor st. W. Toronto 4

Brennan, Terry J., 824 Brookfield Rd., Ottawa 8.

Bright, Richard D., 2512 Regina St., Ottawa.

Brockman, A. M., Huntingdon, P.Q.

Brockway, Dr, Robert W. Ass. Prof., Brandon College, Brandon, Manitoba.

Brooker, Miss Lynda, 109 Gore' St., Kingston, Ont.

Brooks, Wm. H., Room 103 St. John's College, Winnipeg 19

Brown, George W., 23 Lascelles Blvd., Apt. 702 . Toronto 7.

Brown, Gerald R., 1122 Lanark St., Winnipeg 9

Brown, J. A., 13428 Marine Drive, White Rock, B.C.

Brown, L. H. 373 Echo Drive, Ottawa.

Brown, Peter L., The Inst of Historical Research Senate House, Univ. of London, Malet St., London W.C. 1, England.

Brown, Robert Craig, Dept. of History, Univ. of Toronto, Toronto 5.

Browne, Peter L., 42 Scarborough Cresc., Scarborough, Ont. 
Bruchési, Claude, 4660, Chemin de la Reine-Marie, Suite 26, Montréal 26.

Bruchési, S. E. Jean, Ambassade du Canada, Bartolomé Mitré 478, BuenosAires, Argentine.

Brunet, Michel, Faculté des Lettres, Univ. de Montréal, Montréal.

Bryant, J. W., Box 87 . Smithers, B.C.

Buckmaster, Miss Hilda, 74 Henry St., Toronto 2B.

Bumstead, John M., History Dept. Simon Fraser Univ. Burnaby 2, B.C.

Burford, W. T., Box 64, Terminal "A", Ottawa.

Burgess, Miss Lois M., 67 Grove Ave., Ottawa.

Burke, Frank E., c/o Men's Residence, Carleton Univ., Ottawa.

Burkholder, E. L., C.B.C. 140 Wellington St., Ottawa.

Burroughs, Dr. Peter, DaIhousie Univ., Halifax, N.S.

Burrows, Mrs. D. R., 25 Hogan St., Ottawa 5 .

Burt, A. L., 1539 East River Terrace, Minneapolis 14.

Bush, E. F., Public Archives of Canada, Ottawa.

Buss, Dan, Box 75, Frobisher Bay, N.W.T

Buxton, Geo., 2063 KnightsRoad, Ottawa.

Cabana, Mgr. Georges, Archevếque de Sherbrooke Sherbrooke, P.Q.

Cabeldu, J., 2571 William Ave., North Vancouver, B.C.

Caccamo, Joseph, 435 John St., Port Arthur, Ont.

Cairns, H. Allan C., Dept. of Political Science, Univ. of British Columbia, Vancouver 8.

Cadieux, Lorenzo, Laurentian University, Sudbury, Ont

Cairns, John C., Dept. of History, Univ. of Toronto, Toronto 5.

Calder, Loren, Dept. of History, Waterloo Univ. Col lege, Waterloo, Ont.

Caldwell, Major R.V.B., 530 Dewart St., Oakville, Ont.

Campbell, J. R., Sudbury Mining \& Tech. School, Sudbury, Ont.

Campbell, John P., Dept. of History, McMaster University, Hamilton, Ont.

Canoness Sisters, 210 Kenny St., St. Boniface, Manitoba.

Cappadocia, Ezio, Prof. of History, McMaster Univ. Hamilton, Ont.

Careless, Prof. J. M. S. Dept. of History, Univ. of Toronto, Toronto 5 .

Carmichael, Miss Jean B., Glenagle, Kirk's Ferry, P.Q.
Carrière, Prof. Joseph Médard, Dept. of Romance Languages, Univ. of Virginia, Charlottesville, Virginia.

Carrigan, Owen, Prof. of Hisiory, Xavier College Box 760, Sydney, N.S

Carroll, P. J. Queen Elizabeth, District High School, Box 548, Sioux Lookout Ont.

Carson, K. B., 325 George St., Fredericton, N.B

Carson, Paul, 2 Sultan St. Toronto.

Carty, E. B., 160 Waverley St., Ottawa 4.

Cassidy, Keith, Hillhead, R.R. 7, Lachute, P.Q.

Caty, J. J., Ross Mines, Holtyre, Ont.

Cavanagh, Thomas K., 54 Willard Ave., Ottawa 1. Chapman, J. K., Univ. of New Brunswick, Fredericton, N.B.

Chard, Miss W. David, 1598 Edward St, Halifax, N.S Charlebois, Conrad, $138 \mathrm{Mai}$ sonneuve, Hull, P.Q.

Cherihue, V.R., $3007^{-}$West $37^{\mathrm{th}}$ Ave. Vancouver 13 Choate, Miss Bessie P., 5484 Gilpin St., Burnaby 2 .

Christensen, Ray, $1502-$ $8^{\text {th }}$ St. E., Saskatoon, Sask. Church, H. B., Orangeville, Ont.

Clark, George H., New Toronto Secondary School, $18^{\mathrm{th}}$ St., New Toronto, Ont.

Clark, L. C., Dept. of His tory, Univ. of Manitoba, Winnipeg 19.

Clarke, Jamcs Kevin, 103 Main St. E., Ridgetown, Ont.

Clifford, William, 20 Central Street, Guelph, Ont.

Clippingdale, Richard $\mathbf{T}$. Massey College, Univ, of Toronto, Toronto 5.

Cohen, Dean Maxwell, Faculty of Law, 3644 Peel St., Montreal.

Cole, Douglas L., $1010 \mathrm{Ce}-$ dar, Richland, Washington.

Collins, Paul, 1420 Huron St., London, Ont.

Comeau, Roger, Public Archives of Canada, Ottawa.

Conacher, Prof. J. B., Dept. of History, Univ. of Notre Dame, Notre Dame, Indiana.

Condon, Miss Catherine A. 1993 Lynda Lane, Ottawa 8.

Conway, John S., Dept. of History, Univ. of British Columbia, Vancouver.

Cook, G. Ramsay, Dept. of History, Flavelle House, Univ. of Toronto, Toronto

Cooke, A. C., 1742 Western Parkway, Vancouver 8.
Coolidge, Robert T., Dept. of History, Loyola College, 7141 Sherbrooke St. W. Montreal 28.

Coop, James F., $3812-6^{\text {th }}$ St. S.W., Calgary.

Cooper, John Irwin, McGill Univ., Montreal

Cooper, Robert G., Box 100 Jones House, Fredericton, N.B.

Copeland, George F., 825 Richmond St., London, Ont.

Copp, John Terry, 2261

Hingston Ave., Mont real 28

Cornell, Prof. P. G., Dept. of History, Univ. of Waterloo, Waterloo, Ont.

Corry, Prof. J. A., Queen's U., Kingston, Ont.

Corvese, John, 39 Timberlane Drive, Downsview, Ont.

Coryell, Mrs. Warren R. Leskard, Ont.

Cossette, Rev. Joseph, s.i. Coliège Saint-Ignace, 1700 boul. Henri-Bourassa Montreal.

Coulombe, J. T., 1460 Vitré, Québec 3.

Couse, G. S., Dept. of History, Carleton College, Otcawa.

Cowan, Alan W., 26 Nepean St. Ottawa.

Cowan, Helen I, 6303 Blackburn Court, Baltimore 12 , Maryland.

Cowin, John E., 12195 Ridgeway Crescent, Haney, B.C.

Craig Prof. Gerald M., Dept. of History. Univ. of Toronto, Toronto 5 .

Craig, Paul S., 48 Doonaree Drive, Don Mills, Ont.

Crane, Brian A., c/o Gowling, McTavish, 116 Albert St., Ottawa 4.

Crean, J. G., 161 Forest Hill Road, Toronto 7.

Creighton, Prof. Donaid G. Dept. of History, Univ. of Toronto, Toronto 5.

Cross, L. M., Box 389. Fernie, B.C.

Cross, Michael S., Ass. Prof. of History, Univ. of Alberta, Calgary.

Croteau, Benoit, 92 Desbiens, Amqui, P.Q

Croteau, Lionel, Public Archives of Canada, Ottawa.

Crunican, Rev. Paul, Christ the King College, London, Ont.

Crysdale, Rev. Stewart, 85 St. Clair Ave. E., Toronto

Curric, R. G, S., 2308, Sandhurst Ave., Galgary, Alberta.

D'Allaire, Mlle Micheline, 415 St André, Ottawa.

Dalton, Roy C., Bethel College, 1480 North Sne!ling Ave., St. Paul 4, Minn.

Daly, Gerald M., Iroquois Falls High School, Iroquois Falls, Ont. 
Dandurand, Rév. Marcel, Séminaire, Valleyfield, P.Q.

D'Aquino, T. S., 2715 Ewert Cresc. Prince George, B.C.

Davies, Geoffrey O. B., Univ. of British Columbia, Vancouver.

Davies, R. A., 3468 Melrose Ave., Montreal.

Davis, Prof. Arthur K., Sociology, Univ. of Alberta, Calgary.

Davis, George B., 2033 Comox Street, Vancouver.

Davis, Harold A., 29 KingsBury, Bradford, Mass.

Davis, Jack A., 269 Lockhart Ave., Town of Mount Royal 16, P.Q.

Davis, Natalie Zemon, Dept. of Political Science, Univ. of Toronto, Toronto 5 .

Davison, M., 19 Hurlingham Cr., Don Mills, Ont

Dawson, D. A., General Delivery, Brighton, Ont.

Day, M. A. J., 7536 Davies St., Burnaby 3, B.C.

De la Chevrotière, Gerrald, St. Marc des Carrières, P.Q.

Delisle, George, Public Archives of Canada, Ottawa.

Dendy, John, Men's Graduate Centre, Duke Univ., Durham, N. Carolina.

Dent, D. R. Julian, Dept. of History, Univ. of Toronto, Toronto 5.

Deshaies, Bruno, 7230 Musset, Ville St Michel, Montreal.

Desjardins, Leopold, Scolasticat des Pères Rédemptoristes, Mont St-Joseph, Aylmer Est, P.Q.

Desmarais, J. P. 4542 Beaconsfield Ave., Montreal 28. Deutsch, John J., Economic Council of Canada, P.O. Box 527. Ottawa.

DeVolpi, C. P., The Regency, 3555 Côte des Neiges Rd Montreal 25.

Dewar, Kenneth C., 73 St. George St., Toronto 5.

Dick, T. J. O., 24 Farnham Crescent, Ottawa 7

DiGenova, Jimmy, 6513 StDominique, Montreal.

Dobell, I. B. McCord Museum, McGill Univ., Montreal.

Dobeli, Prof. W. M., Dept. of Political Science, Univ. of Western Ontario, London, Ont.

Dodson, Miss Edna, 216 5550 Cambie St., Vancouver 15 .

Dolman, Mrs. F. J., $\mathbf{4 0 ,}$ 665 N. Azure Road, Richmond, B.C.

Donaldson, E. S., 142 Keefer St., Ottawa 2.

Donnelly, Brian E.. 375 Broadway Ave., Toronto 12.

Dorotich, Daniel, Dept, of Slavonic Studies, Univ. of British Columbia, Vancouver 8 .
Douglas, H. T., 212 Rideau Terrace, Ottawa.

Douglas, R. A., R.R. 2, Stittsville, Ont.

Downie, F. Philip, 302 Dorchester Road S., Niagara Falls, Ont.

Draper, H. L., 11935 Acadia Crescent, Haney, B.C.

Drey, Walter, Inc., 257 Fourth Ave., New York 10, N.Y.

Drouin, Gabriel, Institut Généalogique Drouin, 4184 rue St-Denis, Montréal.

Dubuc, Mlle Eugénie, 499 rue de la Morenie, Saint Boniface, Manitoba.

Dumas, Paul E., Public Archives of Canada, Ottawa.

Dunn, Charles W., 1420 West 1.1th Ave., Vancouver 9.

Dunn, Rev, Edward $T$. Mc-Quaid Jesuit High School, 1800 Clinton Ave., Rochester 18 N.Y.

Dunphy, Sister M. Alexandra, R.S.M., 12 Arlington St. Boston, Mass. 02116.

Dunton, A. D., President Carleton Univ., Ottawa.

Duplessis, André," 2041, rue Dézery, Montréal 4.

Durber, 'Paul, 4741' Southlawn Drive, Burnaby 2, B.C.

Dutton, Miss D. M., Box 209. Lennoxville, P.Q.

Eagan, William E., Fanshawe Park Road, R.R. 5, London, Ont.

Eager, Miss Evelyn, Dept. of Economics \& Political Science, Lakehead Univ., Port Arthur, Ont.

Eagle, John A., 39 Raglan St., Toronto 10.

Easterbrook, Prof. W. T. Dept. of Political Economy Univ. of Toronto, Toronto 5.

Eastman, S. Mack, 203 "The Prince Charles", 2776 Pine St., Vancouver ' 9 .

Eaton, R. S., 35 Foothills Drive, Ottawa 6.

Eccles, W. J., Univ, of Toronto, Toronto 5 .

Edwards, Clifford G., 14324 - 92A Ave., Edmonton, Alberta.

Edwards, Miss Nina L., 128 St. James St., London, Ont.

Edwards, W. A., 4384 Sherbrooke St. W., Westmount, Montreal.

Elder, A. Jean, Dept. of History. Univ. of British Columbia, Vancouver 8.

Elhard, Gordon E., 69 Capri Ave., Calgary, Älberta.

Elliott, J. C., 58 Kilbarry Cresc., Ottawa 7

Elmer, G. Lloyd, Macmillan Co. of Canada, 70 Bond St., Toronto 2.

Ely, Marvin, R.F.D.2, Lyme, Connecticut.

Emerson, Miss Margaret A., 26B Summer Road, London, Ont.
Emery, George, 2985 West 14th Ave., Vancouver 8.

Erskine, F. S., 42 Glendale Avenue, Deep River, Ont.

Evans, J. A. S., Dept. of History, McMaster Univ. Hamilton, Ont.

Evans, Roger A., 7131 Elliots Street, Halifax, N.S.

Fairley, Mrs. M. A., 90 Willcocks St., Toronto.

Falconer, Miss Joan M., 400 Wellington St., Ingersoll, Ont.

Farcnholtz, W., Box 192, Abbotsford, B.C.

Farenholtz, Mrs. W., Box 192, Abbotsford, B.C̈. Box

Farr, Dr. David M. L., Carleton Univ., Ottawa.

Farrell, Dr. John' K. A, 1616 Ouellette Ave., Windsor, Ont.

Faucher, Albert, Univ. Laval, Quebec, P.Q.

Ferguson, Dr. W. K., 1061 Waterloo St., London, Ont.

Fergusson, C. Bruce, Public Archives of Nova Scotia, Halifax, N.S.

Ferneyhough, Miss Beatrice 2061 St. Luke St., Montrea

Fieldhouse, Prof. H. N., McGill Univ., Montreal.'

Fiketich, S. P., 1010 Bruce Street, Port Alberni, B.C.

Fingland, F. B., 309 Stewart St., Ottawa.

Firestone, Dr. O. J., 375 Minto Place, Rockcliffe, Park, Ottawa 2.

Firth, Miss Edith G., Toronto Public Library, Toronto $2 \mathrm{~B}$.

Fizzard, Garfield, Memorial Univ; of Newfoundland, St. John's, Nfld.

Flemington, Frank, The RyerSon Press, 299 Queen St., W. Toronto $2 \mathrm{~B}$

Forgarty, Brian, 4166 West 10th Ave., Vancouver 8.

Foley, Prof.' Allan R., Dept. of History, Dartmouth College, Hanover, N.H.

Forsey, E. A., 56 Sparks St., Ottawa 4.

Forster, Donald, Dept. of Political Economy, 100 St. George St., Toronto 5.

Forsyth, Mrs. R. L., 6451 Marine Drive, Burnaby 3 , B.C.

Foster, John W., Dept. of History, Univ. of Saskatchewan, Regina.

Fox. Prof. Paul W., Dept. of Political Science, Univ. of Toronto, Toronto 5 .

Foyster, Kenneth, 257 East 27 St., Hamilton, Ont.

Fregault, Dr. Guy, 586, avenue Mont-Marie, Québec 10.

French, Goldwin, McMaster Univ., Hamilton, Ont.

Friesen, William, 711 Veaverbrook St., Winnipeg 19.

Froats, Cecil S., 22 Trinity Cres., Wondsworth S.W., 17, London, U.K. 
Fuller, G. H., Red Lake District High' School, Box 332, Red Lake, Ont.

Gagnon, Serge, Collège de Sainte-Anne, Ville La Pocatière, Kamouraska, P.Q.

Galarneau, Claude, Univ. Laval, Québec.

Galbraith, John S., Chancellor's Office, Univ. of California, San Diego, La Jolla, California.

Gallagher, Paul, 3465 Durocher, Montreal 18.

Gammell, H. G., 597 Lake Shore Road, Beaconsfield, P.Q.

Garner, John, 2005 Norway Crescent, Ottawa 8.

Gates, Mrs. Paul W., Dept. of History, Comell Univ., Gerson, J. J., Dept. of History, Univ, of Toronto, Toronto 5.

Gerwing, Rev. A. J., O.S.B., St. Peter's Abbey, Muenster, Sask.

Gibbard, John E., 7607 Yukon St., Vancouver 15.

Gibbons, Alan O., 318 Lisgar Road, Ottawa 2 .

Gibeault, Jacques, 383 Champlain, Hawkesbury, Ont.

Gibson, Frederick W. Queen's Univ., Kingston, Ont.

Gibson, Dr. James A., Brook Univ. St., Catherines, Ont.

Gifford, Miss Hilda, Carleton Univ., Ottawa.

Giguère, Rev. Father G E. 25 Jarry Ouest, Montreal 11.

Gilbert, A. D., Box 340, Capreol, Ont.

Gilbert, James M., 257 Colborne St., Port Stanley, Ont.

Gill, Alan F., Trinity College, Toronto.

Gillies, J. H., Box 509, Unity, Sask.

Gilpin, W. J., 501 Delmar, Pointe Claire, P.Q.

Girard, Miss Charlotte, No. 2, 2451 Beresford St., Victoria, B.C.

Gladstone, A., 4967 Kent Ave., Montreal 29, P.Q.

Glashan, A. A., 6300 Côte St. Luc Road, Montreal 29. Glass, John R., Hartland High School,' Hartland, High

Glass, Ogden, Bishop's Univ., Lennoxville, P.Q.

Glassford, Mrs. Ross, 121 Macpherson Ave., Toronto 5.

Glerum, Mrs. C., 11 Oakburn Cresc., Willowdale, Ont.

Glover, Richard G., National Museum of Canada, Ottawa 4.

Gluek, A. C., History Dept. Michigan State Univ., Eas Lansing, Mich.

Godfrey, William G., St. Jerome's College Residence, Univ. of Waterloo, Waterloo, Ont.
Goldenberg, H. Carl, 804 Dominion Square Building, Montreal.

Good, Vera M., Inspector of Schools, 279 Weber St. N., Waterloo, Ont.

Goodsell, James Nelson, 184 Village Street, Millis, Mass. 02054.

Gordon, George, 2415 West 4th Ave., Vancouver 9.

Gordon, H. Scott, Carleton Univ., Ottawa.

Gordon, Michael, 290 McEwen Drive, LaSalle Park, Kingston, Ont.

Gordon, Robert S., Public Archives of Canada, Ottawa.

Gorrell, J. D., 17 Briarwood Avenue, Port Credit, Ont.

Gosselin, Rev. Père JeanLouis, Petit Séminaire, Chemin Carson R.R. 1, Ottawa.

Gouin, Jacques, 59 Prarand Place, Hull, P.Q.

Goulson, Cary F., Univ. of Victoria, Victoria, B.C.

Gow, Mrs. Jean D., 330 Metcalfe St., Ottawa.

Gowen, Robert Joseph, 2829 Kendale Drive, Toledo 6, Ohio.

Graham, Miss Jane E., Dept. of History, Mount Allison Univ., Sackville, N.S.

Graham, W. R., Dept. of History, Univ. of Saskatchewan Saskatoon.

Granatstein, J. L., \& Tormey St., Ottawa.

Grant, John Webster, Emmanuel College, Toronto 5. Grantham, Ronald, Editorial Dept., Ottawa Citizen, Ottawa.

Gray, John, 60 Woodlawn Ave. W. Toronto.

Gray, Paul A. B., 90 Neilson Drive, Etobicoke, Ont

Griezic, Foster J. K., 1124 Meadowlands Drive, Ottawa.

Griffiths, Miss N. E. S., 13 Morris St., Ottawa.

Grylls, John D., Lakefield, Ont.

Guillet, Edwin C., 736 O'Connor Drive, Toronto 6.

Guilmette, Pierre, 852 ave. Chanoine-Scott Sud, Québec 10 .

Guimond, Lionel, Univ. d'Ottawa, Ottawa.

Gundy, H. Pearson, 44 Strathcona Crescent, Kingston, Ont.

Gunn, Gertrude E., 13 Spruce Terrace, Fredericton, N.B.

Hagar, F. A., Dept, of History, Memorial Univ. of Newfoundland, St. John's.

Haigh, David R., Box 494, Maple Creek, Sask.

Hale, Richard W., Archivist of the Commonwealth, State House, Boston 33, Mass.

Hall, Miss Elsie I. N., Federal School Teacher, Discovery, N.W.T.
Hall, H. M., Box 512, Gull Lake, Sask

Hall, Keith J., 84 Beaconsfield Court, Beaconsfield, P.Q.

Haliet, Miss Mary E. William Goodenough House, Mícklenburgh Square, London, W.C. 1. England.

Halpenny, Miss Frances G., Univ. of Toronto Press, Toronto 5.

Hamelin, Marcel, 679 avenue Routhier, Ste-Foy, P.Q.

Hamil, Fred C., 831 Madison Ave., Birmingham, Michigan.

Hamilton, Miss Leone, Bawating Collegiate \& Voc. School, 16 Second Line East Sault Ste Marie, Ont.

Hamilton, W. B. Dept. of History, Duke Univ., Durham, N.C.

Hamilton, w. B., 41 Elmwood Ave. East, London, Ont.

Hands, D. T. Head of History Dept. Port Credit Secondary School, Port Credit, Ont.

Hanna, William B., 19 Relmar Road, Toronto.

Hanrahan, Rev. T. J., St. Mark's College, 5960 Chancellor Blvd Vancouver 8.

Hardy, Rev. Lucien, College St-Boniface, St-Boniface, Manitoba.

Hareven, Tamara K., Dept. of History Dalhousie Univ., Halifax, N.S.

Harris, D. F., 1020 Waterloo St.. London, Ont.

Harris, Dr. R. I. 609 Medical Arts Building, St. George St. George St. at Bloor, Toronto.

Harris, F, J., 6754 - 152 St. R.R. 14, North Surrey,

Harris, Leslis, Memorial Univ. of Newfoundland, St. John's.

Harris, R. C., c/o The Geography Dept., Sidney Smith Hall Univ. of Toronto, Toronto 5 .

Harrisson, Prof. Eric, 505 Annandale, Kingston, Ont.

Harvey, D. C., 1550 Larch St., Halifax, N.S.

Hastings, Edward, 46 Norman St., Stratford, Ont.

Hatch, Fred John, 165 Camelia Ave., Ottawa 7.

Hay, Miss Mary, 87 Elmwood Ave. East, London, Ont.

Healey, Rev. J. E., St. Mary's Univ., Halifax, N.S.

Hecht, Mrs. I. W., 11230 S. W. Collina Avenue, Portland, Oregon 97219.

Heggie, Miss Grace F., 477 Warden Ave., Scarborough, Ont.

Heick, W. H., c/o Dept. of History, Waterloo Univ. College, Waterloo Lutheran Univ., Waterloo, Ont. 
Heisler, J. P., Public Archives of Canada, Ottawa.

Held, Charles H., 5237 Steadman, Dearborn, Michigan. Henderson, George, Wilton, Ont.

Herridge, Herbert W., M.P., House of Commons, Room 648 S, Ottawa.

Hertman, Prof. Lewis, Dept. of History, York Univ., Toronto 12 .

Hetherington, Richard E., 378 Willard Ave., Toronto' 9.

Hett, Robert, Dept. of History, Univ. of Alberta, Edmonton.

Hewlitt, Alexander P., 25 Greenbrae Circuit, Scarborough, Ont.

Heymann, Dr. F. G., Prof. of History, Univ. of Alberta, Calgary.

Higgins, Gilbert E., 2 Exeter Ave., St. John's.

Higgs, Bryon C., Grandview Lodge, McKellar, Ont.

Hilborn, Gordon W.. 329 Cunningham Ave., Ottawa 8.

Hilborn, Dr. K. H. W., Dept. of History, Univ. of Western Ontario, Univ. College, London.

Hill, Lorne, 1636 Gerrard St. East, Toronto 8.

Hillier, Charles, P.O. Box 121, Bonavista, Newioundland.

Hislop, Miss Edna, 18 Glen Manor Drive, Toronto 13.

Hitchins, F. H., 82 Huron St., London, Ont.

Hitsman, J. Mackay, Dept. of National Defence, Ottawa.

Hodgins, Bruce W., 7 Engleburn Place, Peterborough, Ont.

Hodgson, G. R. B., 199 Roehampton Ave., Toronto 12.

Hoffman, Peter, 39 Belholme Ave., Brantford, Ont.

Holloway, Edward B., Social Studies Dept., Atlantic Christian College, Wilson, North Carolina 27893.

Holmes, Miss Janet, 108 Isabella Street, Toronto 5 .

Holmes, John W., Can. Institute of International Affairs, 230 Bloor St. West, Toronto 5.

Home, Mrs. M. S., Dept. of History, Bishop's Univ., Lennoxville, P.Q.

Hood, A. D., 40 Long Crescent, Toronto 13.

Horn, Michiel S., 1228 Johnson St., Victoria, B.C

Horn, Norman E., 387 Sunnyside Ave., Toronto 13.

Horrall, S., 57 First Avenue, Ottawa 1 .

Hotchkiss, Ronald A., 312 Frederick Avenue, Peterborough, Ont.

Houston, Miss M. Jean, Univ. of Toronto Press, Toronto
Howard, S. W., Head of History Dept., Crestwood Secondary School, Box 479, Peterborough, Ont.

Howel, B. A., 2321 Windsor Road, Oak Bay, Victoria, B.C.

Hsieh, Pei-chih, Univ. of Sask., Regina Campus, Re-

Hubbard, Robert H., 54 Roxborough Apts., Laurier Ave. W., Ottawa 4.

Hull, Prof. W. H. N., Dept. of Politics, Brock Univ., St. Catharines, Ont

Humenuk, Stanley, 824 Murrow Court, Lawrence, Kansas 66044 .

Humphreys, John, 304 O'Connor St., Ottawa.

Humphries, Charles W., Dept. of History, Univ, of British Columbia, Vancouver 8.

Hunt, Wm. Claude, 443 Kirkwood Road, Kingston, Ont Hunter, T.M., 29 Birch Ave., Ottawa 7 .

Hutchins, Peter W., 3455 Stanley St., Montreal.

Huyda, Richard J., 157 Mossdale Ave., Winnipeg 15.

Hyatt, A. M. J., 926 William St., London, Ont.

Hyde, Mrs. J. Reid, Como, P.Q.

Hyman, Barry Earl, Student's Memorial Union, Queen's Univ., Kingston, Ont.

Igartua,, Joseph E., Ch. 5504 Pavillon Lemieux, Côté Universitaire, Québec 10.

Imrie, Ian G., 276 Nelson St., Ottawa 2.

Inch, Murray A., 57 Bayswater Ave., Ottawa.

Inglis, Douglas J., 82 Redan St., St. Thomas, Ont.

Ingram, George C., 230 Gloucester, Ottawa.

Ireland, Willard E.. 132 Moss St., Victoria, B.C.

Jaenen, Comelius J., 254 Belvedere St., Winnipeg 12

Jain, Mrs. Geneviève, Glengarry Place, Fredericton, N.B.

Jameson, Miss Sheilagh S. 2736 Cochrane Road, Calgary.

Janidlo. Dan, 7425 Chambord St., Montreal 35.

Janitsch. Mme M. D.. 1115 avenue Laurentide, Québec 6.

Jensen, Rev. Vincent, S.J., St. Paul's College, Winnipeg.

Johannessen, D. I., No. 211 3820 Shelbourne St., Victoria, B.C.

Johnson, Arthur, Box 5880 St. John's, Nfld

Johnson, F. H., 4746 W. 6th Ave., Vancouver 8

Johnson, J. K., Public Archives of Canada, Ottawa 2.

Johnson. Miss P. M., Nanaimo Senior High School, Nanaimo, B.C.

Johnston, C. M., McMaster Univ., Hamitton, Ont.
Johnston, D. Lukin, 1331 Marine Building, Vancouver 1 .

Johnston, Hugh J., Apt. 304, 112 Arbour Glen Cres., London, Ont.

Johnston, J. G., Northern Ontario Building Toronto 2

Johnston, W. S., R.R. 2, Mitchell, Ont.

Johnston, Mrs. Wyndham, 5 Newholm Road, Toronto 8.

Johnstone, R. N., Cobourg East High School, Cobourg, Ont.

Jones, Bryan, The Lakefield Preparatory School, "The Grove", Lakefield, Ont.

Jones, Mrs. D. Wendy, 111 Wurtemburg St., Ottawa 2. Jones, Eloise, M.P., House of Commons, Ottawa.

Jones, Elwood H., Public Archives of Canada, Ottawa.

Jones, R. Bruce, 1827 Westen Drive, Port Coquitlam, B.C.

Jones, Robert L., Marietta College, Marietta, Ohio.

Juble, John, McKay Senior School, Fielen Avenue, Port Colborne, Ont.

Juras, John E., 7509 Champagneur Ave., Montreal 15.

Kage, Dr. Joseph, 4221 Esplanade Ave., Montreal.

Kautz, Arthur P., History Dept., Valparaison Univ. Valparaiso, Indiana.

Kay, Z., Univ. of Sask. Regina Campus, Regina.

Kear, A. R." La Nouvelle Maison des Etudiants, l'Université Laval, Québec 10.

Kendall, John Charles. 340 Metcalfe St., Ottawa 4

Kendle, J. E., Dept. of History, St. John's College, Univ. of Manitoba, Winnipeg 19.

Kennedy, Dr. Philip M. Dept. of History, Univ. of Portland, Portland 3, Oregon.

Keppel-Jones, A. M., Dept. of History, Queen's Univ., Kingston, Ont

Kernoham, Hugh A., Keswick Public School, Keswick, Ont.

Kerr, Prof. D. G. G., 1201 Richmond St., London, Ont.

Kesterton, W. H., Carleton Univ,, Ottawa.

Kilbourn, W. M. 67 Glen Road, Toronto 5 .

King, Peter J., Asst. Prof. of History, Carleton Univ., Ottawa.

King, Roy C., 266 Islington Ave., Islington, Ont.

Kirkwood, D. H., P.O. Box 247, Preston, Ont.

Kitchin, G. Wm., 104 Preston Drive, Nashwaaksis, N.B.

Kitzan, Lawrence, Univ. of Saskatchewan, Saskatoon.

Klassen, Henry C. 2368 Queen St. East, Toronto 13 . 
Klysh, Father Mirone R.. St. Andrew's College, Winnipeg 19

Knowles, David C., 14 Orrin Ave., Ottawa.

Koerner, Walter C., 1203 Matthews Ave., Vancouver.

Koester, C. B., 87 Angus Cresc., Regina, Saskatchewan.

Kolesar, L. F., 244 St. Andre, Gatineau, P.o.

Kornacker, Robert J., St. Jude's School, P.O.' Box 309, LaSalle, Ont.

Kubicek, Robert V., Dept. of History, Univ. of British Columbia, Vancouver 8

Laberee, Waymer S., 1350 Kingston St., Sherbrooke, P.Q.

Laberge, Dr. E. P., Box 155 Ottawa 2.

LaClare, Leo, Public Archives of Canada, Ottawa.

Lacour-Gayet, Robert, 44, rue Jean Goujon (VIII') Paris, France.

L'Allier, Rev. Paul, Collège Jean-de-Bréboeuf, 5625 rue DeCelles, Montreal.

Lamarsh, Peter, Airdrie, Alberta

Lamb, Dr. W. Kaye, Dominion Archivist, Public Archives of Canada, Ottawa.

Lambert, Hon. Norman P., The Senate, Ottawa.

Lambi, Ivo N., Univ. of Sask., Saskatoon, Sask.

Lamy, Miss Thérèse, no. 6, Mount Pleasant Ave., Hudson, P.Q.

Lancaster, W. D., History Dept., West Elgin District High School, West Lorne, Ont.

Landry, Rev. Thomas M. 2490 avenue Royale, Courville, Québec 5.

Lankin, F., 229 West St. N., Orillia, Ont

LaPierre, Laurier L., 75 Chesterfield Ave., Montreal 6.

Larratt-Smith, Mark H., 3955 Ramezay Ave., Montreal 6.

LaTerreur, Marc, Dictionnaire biographique du Canada Bureau du directeur de la recherche, 201 , rue $\mathrm{Mc}$ -

Leod, Ottawa.
Lawn, Robert J., 1814 Playfair Drive, Ottawa.

Lawrence, J. C., Dept. of History, Univ. of B.C. Vancouver 8.

Leach, Barry A., "Tides Ending", 15492 - 40th Avenue. R.R. 1, Cloverdale, B.C.

Leach, Dr. Hamish A., Dept. of History, Univ. of Detroit, 4001 ' W. McNicholas Roit, 4001 Detroit, Michigan Road,

LeBlanc, André, 69 Prudhomme, Hull, P.Q.

LeBlanc, Andre E., $7978 \mathrm{Ba}$ ribeau, Ville LaSalle, Montreal.

LeBlanc, Emery, 11,445 Jean Massé, Montreal 9.
LeBrun, John L., 9315 Lincoln Dr., Northfield, Ohio 44067.

Leddy, Dr. J. F., President, Univ. of Windsor, Windsor Ont.

Lee, Leopold S., P.O. Box 1020, Cowansville, P.O

Leeder, T. J., 1854 Main St. W., Hamilton, Ont.

Lefebvre, J. J., Archiviste Palais de Justice, Montréal 1.

Lefort, André, 3075 Maplewood Avenue, Montréal 26

LeFrancois, J.-J., 273, avenue Wolfe, Levis, P.Q.

Leith, James A., Dept. of History, Queen's Univ. Kingston, Ont.

Leland, Miss Marine, Smith College, Northampton, Mas sachusetts.

Leland, Wallace E., 1216 Royal York Road, Isling. ton, Ont.

LePine, Rev. John J., Cam pion Callege, Regina, Saskatchewan.

Lermer, Arthur, Sir George Williams Univ., Montreal.

Leskiw, Russell J., Medicine Hatt Division No, 4, 147 Sixth St., S.E., Medicine Hatt, Alberta.

Leslie, J. W., 1716 Springland St., Montreal 20.

L'Esperance, Mrs. Jean L.' 3663 Lorne Crescent, Montreal.

Letourneau, Denis, No. 3 , $185^{1 / 2}$ avenue Provencher St. Boniface 6.

Levasseur, Edgar, 45, rue Rideau, Ottawa 2.

Lévesque, Ulric, Mont-Carmel, Co. Kamouraska, P.Q

Levine, Prof. A. L. Dept. of Economics a Poltical Brience, Univ. of Ner

Lewis, Bruce, 315 Rushton Road, Toronto 10

Lewis, Paul E., 161 Sherwood St., Kitchener, Ont

Lightfoot, Gílbert W., 27 Northpark Road, Barrie Ont.

Lingard, C. C., 29 Mohawk Crescent, Qualicun, Ottawa 6.

Little, Adams S., M. D. Univ. of Alberta Hospital Edmonton, Alberta.

Lloyd, Trevor, 73 St. George St., Toronto.

Loft, A. E., 2111 Allenby St., Victoria, B.C.

Lofthouse, Robert G., 124 Park Road N., Oshawa, Ont.

Loggie, Miss Isabel, 1114-87 Avenue, Edmonton, Alberta.

Long, Mrs. Ernest E., 17 Lytton Blvd. Toronto 12

Longarini, David, 256 Hazel St., Sudbury, Ont.

Longley, Prof. R. S.. Acadia Univ., Wolfville, N.S.
Loosley, Miss Elizabeth, Dictionary of Canadian Bio. Univ. of Toronto Press, Toronto 5.

Loudon, Donald E., 217 East 12 th St., New York, N.Y. Lovekin, James, 12 Pine Ridge Drive, Scarborough, Ont.

Lovell, Colin Rhys., Univ. of Southern California, Los Angeles 7, California.

Luxton, D'Árcy, c/o Martin \& Martin, 15 King St. W., Hamilton, Ont.

Luxton, Miss Eleanor G., 616 - 13th Ave. S.W., Calgary, Alberta.

Lynch-Staunton, John, 3482 Holton Ave., Montreal 6

Lyne, Daniel Colman, St. Patricks College, Ottawa 1. McCaffrey, R. Bruce, 80 Grandravine Drive, Downsview, Ont.

McCartney, J. Edward, 751 George 'St. North, Peter borough, Ont.

McCartney, James R., 53 St. Ives Crescent, Toronto 12.

McCloy, T. R., 1941 Grand Oaks Drive,' Calgary, Alberta.

McClure, William H., P.O Box 543, Saginaw, Michigan.

McCready, Prof. H. W., McMaster Univ., Hamilton, Ont.

McCullough, Edward E., 1441 Drummond St., Montreal

McCusker, John J., Dept. of History, St. Francis Xavie Univ., Antigonish, N.S.

McCutcheon, M. Wallace, 522 University Ave., Toron to 2.

McDermott, Michael, International Labour Affairs, Sir Wilfrid Laurier Bldg. Ottawa 4.

MacDonald, Rev, David s. H., Alberton, Prince Edward Island.

Macdonald, W. A., Director, Historical Section, C.B.C. Historical Section, C.B.C., "A", Ottawa 2.

MacDónell, Rev. Malcolm St. Francis Xavier Univ. Antigonish. N.S.

McDougall, D. J., 17 Tranby Ave., Toronto '5.

MacDougall, Rev. H. A., St. Patrick's College, Echo Drive, Ottawa.

McDougall, Hugh, Head of History Dept., St. Michael's College School, $1515 \mathrm{Ba}$ thurst St., Toronto 10.

McDougall,' Miss Mary C. c/o Miss J. S. McDougall, 581 Jarvis St., Toronto 5.

McErvel, Miss Judith, Archives Office, The T. Eaton Co. Ltd., Toronto 1.

McEwen. Dr. John M., Dept. of History, Univ. of Manitoba, Winnipeg 13 .

McEwen, Edward H., 5565 Chester St., Vancouver 15 .

McFadden, Miss Eileen $M$. 25 - 20th Street, Brandon, Manitoba. 
McFarland, Miss Kaye, Box 445, Napanee, Ont.

MacFarlane, Dr. R. $O$., Carleton Univ., Ottawa. McGee, Frank, 20 Chatfield Drive, Don Mills, Ont.

MeGillivray, A., 32 Fulton Ave. Toronto 6.

McGillivray, R., 6030 Lanark St. Vancouver 15.

MacǴowan, S. D., 820 Levis Ave., Quebec 6.

McGuinness, Frend, Box 60 , Medicine Hat, Alberta.

McInnes, Edgar, York Univ. Toronto.

MacIntosh, Prof. Alan, Dept. of History, Acadia Univ., Wolfville, N.S.

McIntosh, Hilton A., 111 Wurtemburg St., Ottawa 2. MacIver, A. F., Box 98, Churchill, Manitoba.

McKague, Ormond K., 2904 Grant Road, Regina, Saskatchewan.

Mackay, Miss Lovisa, 46 Health Road, Montreal 9.

MacKay, R. A., Carleton Univ., Ottawa.

McKellar, Peter, P.O. Box 5, Spirit River, Alberta.

MacKenzie, Miss Hazel C. P.O. Box 104, Baddeck, N.S.

Mackenzie, P. Hamilton, 656 Belmont Ave., Outremont, P.Q.

Mackenzie, Br. R. L., Regina Central High School, Corner Brook, Nfld.

Mackinnon, Dr. Frank, Prince of Wales College, Charlottetown, PE.I.

MacKinnon, Rev. Hugh, s.j. Dept. of History, Univ. of Waterloo, Waterloo, Ont.

McKinnon, J. A., Box 1510, Quesnel, B.C.

MacKirdy, K. A., Dept. of History, Univ. of WaterHistory, Univ. of

loo, Waterloo, Ont.
McKnight, Alan, P.o. Box 358, Shilo, Manitoba.

McLachlan, Robert T., Williamstown, Ont.

McLaughlin, John P., 124 St. James St., London, Ont.

McLaughlin, Mrs. Lorrie, 981

McLaughlin, Mrs. Lorrie, 981
Main St. West, Hamilton, Ont.

MacLean, A. Harry, Acadia Univ, Wolfville, N.S.

McLean Mrs. Barbara, Box 427, Énugu, Eastern Nigeria.

MacLean, Duart A., 5435 Aderley Road, R.R. 5, Victoria, B.C

MacLean, Guy R., Dalhousie Univ., Dept. of History Halifax, N.S.

MacLean, Neil, 3483 Warren St., Halifax, N.S.

MacLean, R.A., Box 99, St. Francis Xavier Univ., Antigonish, N.S.

McLeod Keith A., 2015 7th St. E., Saskatoon, Saskatchewan.

MacLeod, Mrs. Margaret A. 138 Maryland St., Winnipeg, Manitoba.
McNaught, Prof. K. W. Dept. of History, Univ. of Toronto, Toronto 5.

McNeal, Prof. Robert $H$. Dept. of History, Univ. of Toronto, Toronto 5.

McNicol, Miss Helen K., No. 5, 1708 Boxen Road, Nanaimo, B.C.

MacNutt, Prof. W. S., Univ. of New Brunswick, Fredericton, N.B.

MacQuarrie, H. N., House of Commons, Ottawa, Ont.

MacVean, Rev. Wm. C., College of Emmanual \& $\mathrm{St}$. Chad, Emmanuel College, Saskatoon.

McVey, Kenneth A., Box 340, Grandview, Manitoba.

Machum, L. A., Mount Allison Univ., Sackville, N.B.

Magee, Mrs. Marion, Univ. of Toronto Press, Univ. of Toronto, Toronto 5.

Mahon, Henry MacLeod, Box 1, South Duxbury, Mass.

Major, T. G., "The Juliana", Apt. 401, 100 Bronson Ave., Ottawa.

Malchelosse, Gerard, 1289. rue de la Visitation, Ste Foye, Québec 10

Manning, Mrs. Helen Taft, Featherbed Lane, Haverford, Penn.

Manning, John, 122 Bessey Hall, Dept. of Humanities, Michigan State Univ., East Michigan State Unir.

Mantha, Hector, 65 Milton, Montreal 18.

Marcellien, Frère, Académie de La Salle, 373 Sussex, Ottawa 2.

March, R., Dept. of Political Science, Carleton Univ. Ottawa.

Marchand Richard, 9151 Chateaubriand, Montt. 11.

Marcinek, Robert, 2935 Linton Road, Ottawa 10.

Marsh, Peter T., Dept. of History, Univ.' of Sackatchewan, Saskatoon.

Marshall, H. S., 30 First St., Oakvilie, Ont.

Martin, J.' L., History Dept. Southwood Sec. School, Southwood Drive, Galt, Ont.

Martin, John L., 157 Grand Ave. South, Gait, Ont.

Martin, Miss Marguerite, 833 Galt Ave., Verdun 19, P.Q

Martini, Dominic R., 6034 Hurteau Montreal "20.

Martyn, John, 299 Old Orchard Grove, Toronto 12 .

Mason, Miss Lorna M,, Kinmount, Ont.

Mast, James H., 107 Byron Road, Pittsburgh, $\mathbf{P a}$

Masters, Donald C., Bishop's Univ., Lennoxvilie, P.Q.

May, Miss Betty, Russell, Ont.

May, C. Kennedy, 36 Castlefrank Road, Toronto 5 .

Mays, H. J., The Crestview Apt. 11, 1100 Hillcrest Ave., Sackville, N.B.
Mealing, S. R., Carleton Univ., Ottawa.

Mercier, P. B., 3500 Gouin Blvd., Montreal 14.

Mère 'Ste Suzanne Marie, Congrégation Notre Dame, 38 Gloucester St., Ottawa 4.

Metcelfe, W. C., Dept. of History, Univ. of Vermont, Burlington, Vermont 05401. Meyer, H. A., 1878 Alberni St., Vancouver.

Mezaks, Janis (John), 193 Woburn Ave., Toronto 12.

Milborne, A. J. B., P.O. Box 248, Knowlton, P.Q

Miles, Miss Helen, 82 South Drive, Toronto 5 .

Miles, John A., 104 Cheltenham Ave., Toronto 12.

Miller, James R., 412 Seventh St. E., Cornwall, Ont.

Miller, T. B., Lakehead College, P.O.'Box 1166, Port Arthur, Ont.

Miller, Warren C., B.Sc., 67 Gladstone Ave., St. Thomas, Ont.

Milligan, Dr. John D., 21 Allenhurst, Buffalo, N.Y. 14214.

Minichiello, John, 2186 E. Pender St., Vancouver 6, B.C.

Miquelon, $D_{\text {., }} 166$ Jameson, Apt. 808, Toronto.

Mitchell, Harvey, Univ. of Alberta, Calgary.

Mitchell, Peter M., Apt. 7D, 542 W. 112th St., New York, N.Y. 10025

Mitchell, Robert A., Nobel, Ont.

Mizener, W. E., Public Archives of Canada, Ottawa 2.

Molyneaux, John L., 14 4 New Copeley Hill, Charlottesville, Virginia 22901.

Monet, Rev. Father Jacques, S.J., 1855 est Rachel, Montreal 34.

Monks, Miss Lois M., 6707 Argyle St., Vancouver 15.

Moore, Miss Evelyn R., Faculty of Education, Univ. of Alberta, Edmonion.

Moore, R. F., 323 Bunnhamthorpe Road, Cooksville, Ont.

Moreau, Raymond, 639 Sunset Ave., Windsor, Ont.

Morley, Wm F. E., 36 Hillendale Ave., Kingston, Ont.

Morren, Douglas, M. A., 484 Evered Ave., Ottawa 3.

Morris, Miss A. Y., 120 Strathcona Ave., Ottawa 1.

Morris, N. Oswald, 18 Fitzgerald Lane, Port of Spain, Trinidad.

Morris, Thomas, No. 7, 8679 Montcalm St., Vancouver 14.

Morrison, H. D., Box 280 , Macdonald College, Ste 
Morrison, Ian W., Dept. of Social Sciences, California State Polytechnic College, San Luis Obispo, California 93401

Morrison, J. C., 86 Aero Drive, Lekeview, S.S. 4 Ottawa

Morton, D. P., c/o New Democratic Party of Ontario, 111/2 Spadina Road. Toronto 4.

Morton, R. E., 1130 Guildwood Blyd., London, Ont

Morton, W. L., Univ. of Manitoba, Winnipeg, Man.

Mui, Hoh-cheung, Dept. of History, Memorial Univ. of Nfld., St. John's.

Muir, Robert, M. P. 107 Shore Road, Sydney Mines, N.S

Muise, D. A., 1038 Brough St., London, Ont.

Mullally, Dr. Emmet J., 21 Melbourne Ave., Westmount, P.O.

Muller, H. N., Dept. of History, Mount Allison Univ. Sackville, N.B.

Muller, Prof, Steven, Dept. of Government, Cornell Univ. Ithaca, New York.

Mulvihili, Rev. D. J., Assumption Univ., Windsor, Ont.

Munro, Dr. John H. A., History Dept., Univ. of British Columbia, Vancouver 8.

Munro, R. D., 150 Herkimer St., Hamilton, Ont.

Munroe, David C. Macdonald College, Ste Anne de Bellevue, P.Q.

Murdoch, Arthur Wm., 458 St. Clement's Ave., Toronto 12.

Murkin, Stewart O., 1095 Jefferson Ave., West Vancouver.

Murray, Alex L., Dept. of History, York Univ., Toronto 12.

Murray, D. K., 7 Maytree Road, Willowdale, Ont

Murray, Miss Jean E., Univ. of Sask., Saskatoon.

Murray, W. D., 3031 - 18th Ave., Regina, Saskatchewan.

Nadeau, Gabriel, Rutland State'Sanatorium, Rutland, Mass.

Naftel, Wm. D., Public Archives of Canada, Ottawa.

Naylor, Ronald P., 330 Brock Ave. North, Montreal 28.

Neatby, Prof. H. B., Dept of History, Carleton Univ., Ottawa.

Neilson, Miss Joan M., 8 Haddon St., Toronto 12

Nelson, Harold I., Dept. of History, Univ. of Toronto, Toronto 3.

Neutel, Walter, Public Archives of Canada, Ottawa.

Newton, Mark A., 3460 Simpson St., Montreal.

Nicholson, Col. G.W.L., 1101 Bronson Ave., Ottawa.
Nicks, John S., $16-46$ Spence St., Regina.

Nicolin Peter, 11406 - 38 St., Edmonton.

Nijhowne, Tilak, Dept. of Economics, Sir George Williams Univ., Montreal 25.

Nish, Cameron, Dept. of History, Collège Militaire Royal, Saint-Jean, P.O.

Nixon, Mrs. R. J., 835 Riverwood Ave., Winnipeg 19.

Noble, C. W., History Dept., Ridley College, St. Catharines, Ont.

Noxton, David B., 32 Chestnut Park, Toronto 5.

O'Connell, Miss Carole Ann, 5240 Trans Island Ave. Montreal 29.

O'Connor, Rev. J. S., C.R. Resurrection College, 2727 King St., E., Kitchener, Ont.

Ogelsby, J. C. M., Dept. of History, Victoria College Victoria, B.C.

Oglesby, Major R. B., 12 Hockley Place, Don Mills. Ont.

O'Hearn, Michael, 366 Shannon Road, Sault Ste Marie Ont.

O'Keefe, Rev. C. B., Loyola College, 7141 Sherbrooke St. West, Montreal 28.

Olineck, Peter, Box 217 . Lanigan, Sask.

Oliver, Michael, 631 Grosvenor Ave., Westmount, P.Q.

Oliver, Peter N., 185 Fairview Ave., Toronto.

Ormsby, Miss Margaret A. Univ. of British Columbia Vancouver.

Ormsby, Wm. G., History Dept., Brock Ưniv., St. Catharines, Ont.

Orr Allan D., 1855 Balsam St., Vancouver 9.

Osborne, K., 200 Hespeler Ave., Winnipeg, Manitoba. Ossman, Albert J., Prof. of Economics and Political Science, Chatham College. Pittsburgh 32, $\mathrm{Pa}$.

Ostiguy, Lt. Col. J. W. National Research Council, Ottawa.

O'Sullivan J. F., 443 Echo Drive, Ottawa 1

Ouellet, Fernand, 6 Kilbarry Cresc., Ottawa.

Outhet, Murray, 513 Court Court St., Aylmer East, P.Q.

Overduin, Henry, P.O. Box 188, Mount Brydges, Ont. Overend, Harold J., 11 Albert St. S., Orillia, Ont. Overton, Dr. R. C., Dept. of History, Middlesex College, Univ. of Western Ontario. London, Ont.

Page, Donald, 108 Isabella St., Toronto 5 .

Page, Robert, 206 Joicey Blvd., Toronto 12.
Panting, Prof. G. E., c/o Dept. of History, Memorial Univ. of Nifd., St. John's.

Parizeau, Gerard, 10 avenue Glencoe, Outremont, Montreal

Park, F. W., Quinta Avenida 808, Apt. 4, Miramar, La Habana, Cuba

Parker, Reg. A., 123 Midland Ave., Beaurepaire, P.Q.

Parsons, Robert W., 287 Airdric Road, Toronto 17.

Paterson, Major J. S., 4 Croxton Road, London, Ont.

Patterson 2nd, Dr. E. Palmer, St. Jerome's College, Waterloo, Ont

Patterson, Frank H., 28 Whitmon Court, Truro, N.S

Patterson, G. H., 165 Grand River St. W. Paris Ont.

Pawluk, Stephen, 570 Rogers Road, Toronto 15.

Pearce, C. W., Ste 405, Rideau St Ottawa 2

Pearce, Rev.' George R., 12 Prefontaine Ave. West, Sainte Agathe des Monts, P.Q.

Pearson, Rt. Hon. L. B. Prime Minister of Canada, East Block, Ottawa.

Pease, William H., Univ. of Alberta, Alberta.

Pech, Stanley Z., Dept. of Slavonic Studies, Univ. of British Columbia, Vancouver 8.

Peel, Bruce, $11047-83$ Ave., Edmonton. Alberta. Pendergast. Rev. R. A., St. Joseph College, Univ. of Alta. Edmonton Campus, Edmonton.

Penlington, Dr. Norman, Dept. of Humanities, Michigan State Univ., East Lansing, Michigan.

Pennefather, Robert S., Dunnville, Ont.

Penner, Peter, P.O. Box 524 Sackville, N.B.

Pentland, H. C., Dept. of Economics. Univ. of Manitoba. Winnipeg.

Pepall, Robert L., 217 Lonsdale Road, Toronto 7.

Père Jean de la Croix, Les Pères Capucins, Cap Rouge, P.Q.

Perepelkin, Peter, R.R. 1 Winlaw, B.C.

Perinbam, Mrs. B. Marie 3620 Quesada St. N. W. Washington, D.C. 20015 .

Perry, Michael J. E., Trinity College School, Port Hope, Ont.

Peters, Dr. Victor. Prof. of History, 86 Westgate, Win-

nipeg. $R$ a y m o n d. 106 Marchmount Road, Toronto.

Phillips, A. T.. 457 Forest Ave., West Kildonan, Winnipeg 4. 
Phillips, Mrs. N. W. F., 227 Gay-Lussac Street, Arvida, P.Q.

Pickersgill, Hon. J. W., House of Commons, Ottawa.

Piepenburg, W. W., Office of the Dean of the Faculty of Arts \& Science, York Univ., 2275 Bayview Ave., Toronto 12

Pierce, Richard A., Asst. Prof. of History, Queen's Univ., Kingston, Ont.

Pigeon, L. P., Univ. of Ottawa Teacher's College, 115 Wilbrod St., Ottawa 2.

Piovesana, Roy, 418 Westview Place, Fort William, Ont.

Pitfield, Michael, Saraguay House, 8432 Gouin Blvd. West, Pierrefonds, P.Q.

Plumpton, John G., Apt. 301, 1 Deauville Lane, Don Mills, Ont.

Poirier, J. C. Noël, 45 St. Paul St., Alexandria, Ont

Polika, Julian, 632 Bathurst St., Toronto.

Pollard, S. L., 10961 Esplanada' Ave., 'Montreal 12 .

Potvin, J. Robert, 87, rue Ontario, Ottawa 4.

Potvin, M. 1'abbé Pascal, Ecole Normale, 53 Déziel, Lévis, P.Q.

Poulin, Claude, 56 Saint Louis, Québec, P.Q.

Pouliot, R. P. Adrien, 14 rue Dauphine, Oué. P.Q. rue Dauphine, Qué. P.Q. Senate, Ottawa, Ont.

Prang, Miss Margaret E., Dept. of History, Univ, of British Columbia, Vancouver.

Preston, Anthony W., Bishop's Univ., Lennoxville, P.Q.

Preston, R. A., Royal Military College, Kingston, Ont. Prideaux, J. M., 122 Beechwood Ave., Willowdale, Ont.

Pritchard, H. D., Pentincton Jr.-Sr. High School, 158 Eckhardt Ave. East, Penticton, B.C.

Pritchard, H. D., PentincThomas St., Toronto 5.

Pryke, Kenneth, Dept. of History, Univ. of Windsor, Windsor, Ont.

Pullen, Rear Adiniral H. F., "Big Hill", Chester Basin, N.S.

Purdy, Judson D., Pickering College, Newmarket, Ont.

Quealey, Rev. F. M., C.S.B., St. Michael's College, Bay and St. Joseph Sts., Toronto 5 , Ont

Raddail, Thomas H., 44 Park St., Liverpool, N.S.

Ralston, H. Keith, 3037 West 28 th Ave., Vancouver 8.

Rasch, Otto, Bahnhofstrasse 5, Marburg Lahn, Germany. Rausch, George J., Jr., 700 Polaris Place, Puliman, Wash. 99163.
Rawlyk, George A., 1941 Oxford St., Halifax, N.S.

Raymond, Raoul, 8960 rue St-Denis, Montreal 11.

Reed, Frederick, 205 - 56 Ave., Lachine, P.Q.

Reford, Eric, 221 St. Sacrament St., Montreal 1

Regehr, T. D., Public Archives of Canada, Ottawa. Reid, John M., 107 Sweetland, Ottawa 2.

Reid, W. Stanford, Dept. of History, Univ. of Guelph, Guelph, Ont.

Renaud, Jules, 622 Carleton St., Cornwall, Ont.

Reynolds, R a l p h, Canada House, London, S.W.L., England.

Richardson, G. W., 24 Downing St., Ottawa. Richardson, John F., 4809 Melrose Ave., Montreal 29. Richer, Mlle Charlotte, 1943 ave. Tweed, Ottawa 8.

Richer, Mlle Yolande, 1943 ave. Tweed, Ottawa 8.

Ridge, Alan D., McGill Univ., Montreal.

Ridout, John, Whidden Hall, McMaster Univ., Hamilton, Ont

Rife, C. W., 1689 Hewitt Ave., St. Paul 4, Minn.

Risk, R. C. B., Faculty of Law Univ. of Toronto Law, Univ.

Ritcey, Walter R., 199 Harcourt Ave., Ottawa 14.

Ritchie, Ronald S., c/o Imperial Oil Ltd., 111 St. Clair Ave. West, Toronto.

Roberts. Lloyd G., 3933 Sefton St., Port Coquitlam, B.C.

Robertson, Rev. Fr. D. F. Box 27, Pangman, Sask Robertson, Mrs. Duncan, 52 Florence St., Kingston, Ont. Robertson, Ian R., Apt. 10, 5171 Sherbrooke St. West, Montreal 28

Robertson R. W. W., Clarke, Irwin \& Co. Ltd., 791 St. Clair Ave. W., Toronto 10

Roby, Yves, 1486 St. Paul Ancienne Lorette, Quebec.

Rodney, Dr. William, c/o Dept. of History, Royal Roads, Victoria, B.C

Roe, Frank G.. 2547 Killarney Road, Cadboro Bay, Victoria, B.C.

Rollit, J. B., Canadian Pacific Railway, Room E 201, Windsor Station, Montreal. Ronan, M. L., Box 258, Little Current, Ont. Rosenberg L. 493 Sherbrooke St. W., Montreal 2 Ross, Sam G., 196 Kamloops St., R.R. 5, Ottawa.

Ross, W. M.. 373 Steveston Highway, Steveston, B.C.

Rothney, Gordon O., Lakehead College of Arts \& Science \& Technology Port Arthur, Ont.

Rotstein, Abraham, Dept. of Political Economy Univ. of Toronto, Toronto.
Rousseau, Jean-Claude, 30 boul. de Varennes, Boucherville, P.Q

Rowe, Prof. J. G., Dept. of History, Huron College, London, Ont.

Roy, Miss Patricia, 218 Queen's Ave., New Westminster, B.C.

Roy, Reg H., 3825 Merriman Drive, Victoria, B.C

Rudkin, David W., 703 Randolph Ave., Windsor, Ont. Rushton, Theodore A.̈ 334
West St. North, Orillia, Ont.

Russ, Chris. J., 128 Stratcona Drive, Town of Mount Royal, Montreal 16.

Russell, Edward C., 516 Blair St., Ottawa.

Rutherford, W. D., Inst, of Iroquoian Studies, $_{\text {P.O }}$ Box 1024, Brantford, Ont.

Ruud, Charles, Lecturer in History, Univ, of Western Ontario, Middlesex College, London, Ont.

Ryan, Miss Janice, 4015 Oxford Ave., Montreal 28. Ryerson, S. B., 84 Gormley Ave., Toronto 7.

Rysyk, R. W., 41 Risdon Ct., Etobicoke, Ont.

Sage, Donald, 2503 W. 37th Ave., Vancouver 13.

Salmon, Dr. $E$. T Dept of History, McMaster Univ., Harnilton, Ont.

Sandblom, H. E. H., 876 Tulane, Sudbury, Ont.

Saucier, Roger, 105F Ave. Fournier, Quebec 10.

Saunders, Robert E., 48 Sonneck Square, Scarborough, Ont.

Savage, Dr. Donald C., 4071 Grand Blvd., Montreal 28.

Savory, Gerald N., Dept. of Univ. Extension, Univ. of British Columbia, Vancouver $B$.

Sawatsky, Aron, $3907-49$ Ave., Lloydminster, Sask.

Sawdon, W. B., P.O. Box 639, Sackville, N.B.

Saywell, John T., York Univ., 2275 Bayview Ave., Toronto 12 .

Schlieper, H. C., 3663 Lorne Crescent, Apt. 1, Montreal.

Schneider, Fred D., Dept. of History, Vanderbilt Univ., Nashville 5, Tenn.

Schoeck, Prof. R. J., Pontifical Inst. of Med.'Studies, St. Michael's College, University of Toronto, Toronto

Schull, Joseph, St. Eustache sur le Lac, Co. of Two Mountains, P.Q.

Schultz, Dr. Harold J., Dept. of History, Stetson Univ. Deland, Florida.

Schurman, D. M., 59 Silver St., Kingston, Ont.

Schuyler, Robert Livingston, 253 Alexander St., Rochester 7. 
Schwarz, George M., Dept. of History Memorial Univ. of Nfld., St. John's.

Scorgie, Miss Karen, 274 Sunview St., Waterloo, Ont.

Scott, Miss Christine M., Time Int'l of Canada Te., 1155 Dorchester B $1 \mathrm{v}$ d. West, Montreal.

Scott, F. R., McGill Univ., Montreal.

Scott, W. R., 1 Brushwood Court, Don Mills, Ont.

Scotvold, John L., No. 1 , 515 - 6 St. N.E., Calgary, Alberta.

Seaborn, R. G., 321 Waverley Ave., Ottawa

Senior, Dr. H., 3438 Prud'homme Ave., Montreal 28

Serio, Miss Ann Marie, 384 Fargo Ave., Buffalo, N.Y. 14213 .

Sessions, Kyle C., Ass. Prof., Huron College, London, Ont.

Sexton, Miss Anne M., 185 College Ave. London Ont.

Shadd, D. T. W., Public Archives of Canada, Ottawa.

Shaffer, A. Louis, Silverthorn Public School, 72 Ewart Ave., Toronto 15.

Sharp, Philip A. 29 Dominion Ave., Kapuskasing. Ont.

Shelton, Dr. W. G., Victoria College, Victoria, B.C

Shields, R. A., Ófice 226 , Univ. of Alberta, Calgary.

Shipton, James F., Pen Lake, P.O., Huntsville, Ont.

Shkwarok, W. F., P.O. Box 314. Castlegar, B.C.

Shoebridge, Tom, Dept. of History, Notre Dame College, Wilcox, Saskatchewan.

Shore, D. A., P.O. Box 585, 100 Mile House, B.C

Siberry, Miss S. K., 750 Coxwell Ave., Toronto 6

Sievwright, Dr. E. C., 1554 Yonge St., Toronto 7.

Simpson, George W., 1015 Osler, Saskatoon, Sảsk.

Sinn, Harry L., 702 South Eighth Ave., Yakima, Washington.

Sissons, J. N., 204 Glenrose Ave., Toronto.

Sister François de Sienne. Congrégation de NotreDame, 38 Gloucester, Ottawa.

Sister Mary Evangelista, Immaculate High School, 211 Bronson Ave., Ottawa.

Sister Mary Jane, St. Joseph High School, Islington, Ont.

Sister Mary Jean, Saint Mary of the Woods College, Saint Mary of the Woods, Indiana.

Sister Mary Liguori, Mount Saint Vincent College, Halifax, N.S.

Sister St. Amelia, C.N.D. 3702 Ste Famille, Montreal 18.
Sister St. Callista, C.N.D., Prof. of History, Mariano polis College, 3647 Peel St., Montreal 2.

Sister St. Mary of Cana, C.N.D., Marianopolis College, 3647 Peel St., Montreal 2.

Sister St. Veronica, Prof. of History, St. Francis Xavier Univ., Antigonish, N.S.

Sister Thérèse Carmel, Mount Saint Vincent College, Halifax, N.S.

Small, Mrs. A. Douglas, 753

Lonsdale Road, Ottawa 7

Smith, Miss Carole Inez, 5019 Batchelor Cres. N. W., Calgary.

Smith, Donald W.. P.O. Box 219, Bridges House, Fredericton. N.B.

Smith, Dr. Dwight L., Dept. of 'History, Miami 'Univ., Oxford, Ohio

Smith, E. F. Schiewick, P.O. Box 126, Hudson Heights, P.Q.

Smith, Gaddis, 110 Filbert St., Hamden, Conn

Smith, Gordon W., 57 Blackburn Ave., Ottawa 2.

Smith, Miss Marion B., Dept. of English, Univ. of Manitoba, Winnipeg

Smith, Peter, 1301 Coal Ave. S.E., Albuquerque, New Mexico.

Smith, R. D. K., 122 Dunsmure Road, Hamilton, On

Smith, S. Denis, 509 Homewood Ave., Peterborough, Ont.

Smith, W. D., Brandon College, Brandon, Manitoba.

Smith, Rev. W. E. L. Queen's Theological

lege, Kingston, Ont.

chives of Canada, Ottawa.

chives of Canada, Ottawa. Gananoque, Ont.

Sniderman, Stephen L., Apt. 610,11 Shallmar Blvd., Toronto 10

Snowdon, Miss Muriel, 299 Riverside Drive, St. Lambert, P.Q.

Soban, S., 1117 Ave. Road, Toronto 12.

Somers, Rev. T. H. B., The Kirk House, Charlottetown, P.E.I.

Somerville, R. B., 38 Dixington Cres., Apt. 103, Weston, Ont.

Spencer, Miss Rita V., 437 Elm St., Saskatoon, Sask. Spencer, Robert A. Dept. of History, Univ. of Toronto History,

Toronto.
Splane, Richard B., 116 Sherwood Drive, Ottawa 3.

Spragge, George W 168 Strathallan Blvd., Toronto 12 .

Spry, Mrs. Graham, 28 Chester St., London, s.W. 1, England.

Stanley, Dean G. F. G., Royal Military College, Kingston, Ont.
Stanley, Robert E., Dept. of History, Ridley College, St. Catharines, Ont.

Stearns, Owen P., 7 Pierce Road, Watertown 72, Mass.

Steele, Ian K., 295 Deer Park Circle, London, Ont.

Stevens, Lt.-Col. G. R., 144 Strathearn Ave., Montreal West.

Stevenson, Andrew A., 4022 Oxford Ave., Montreal.

Stevenson, Hugh A., 101 Weymouth St., Charlottetown, P.E.I.

Stewart, Miss Alice R. Univ. of Maine, Orono, Maine.

Stewart, Prof. John H., Dept. of History, Western Reserve Univ., Cleveland 6, Ohio.

Stockdale, William, Christ the King College, Univ. of Western Ontario London.

Stockton, Alan, 322 Inglewood Ave., Pointe Claire, P.Q.

Stokes, L. D., 3301 St. Paul St., Apt. 401, Baltimore 18, Maryland.

Strachan, R. H., The MacMillan Co. of Canada Ltd. 70 Bond St.. Toronto.

Stratton, Mrs. R. S., Secondary Education Banch, Western Ontario Division, 1071 Waterloo St., London, Ont.

Strikwerda, Earl, Dept. of History, Calvin College, Grand Rapids 6, Michigan.

Strikwerda, Mrs. E., 1231 Franklin St. S.W." Grand Rapids, Michigan 49506.

Stuart, W. H., Little Current High School, Lttle Curent, Ont.

Stubbs, John, 118 Douglas Drive, Toronto 5.

Sugimoto, Howard H., 4009 - 15 Ave. N.E., Seattle 5, Washington.

Sutherland, Prof. J. K., College of Education, Univ of Saskatchewan, Sask

Sutherland, J. Neil, c/o Faculty of Education. Univ. of British Columbia, Vancouver 8.

Swainson, Donald, Dept. of History, Queen's Univ. Kingston, Ont.

Swanick, Dale R., R.R. 3, Uxbridge, Ont.

Swayze, J. F., R.R. 3, King City, Ont.

Swift, Michael, Public Archives of Canada, Ottawa.

Swyers, David V., No. 505 , 1129 Meadowlands Drive, Ottawa 5.

Sylvain, Frère Robert, 2360 Chemin Sainte-Foy, Québec 10.

Symons, T. H. B., President \& Vice Chancellor, Trent Univ., Peterborough, Ont.

Tague, Dr. James A., History Dept., Memorial Univ. of Nfld., St. John's, Nfld.

Talman, J. J. Univ, of Western Ontario, London, Ont. 
Tanchak, E., 133 Mossdale Ave., Winnipeg 15 .

Tancock, K. C., 55 Beverly G. (Hornchurch), Essex, England.

Taylor, Dennis A., 1396 Crescent Road, Lorne Park, Port Credit, Ont.

Taylor, J. Craig, 18A Summerhill Gardens, Toronto 7 Taylor, Miss Mary, Dept. of History, Carleton' Univ., Ottawa.

Taylor, Mrs. Robert F., 27 Warner Road, Grosse Pointe Farms, Michion 48236

Taylor, Robert John, 347

Bartos Drive, Oakville, Ont.

Teakles, Mrs, J. M., 165

Takeway Drive, Rockcliffe, Ont.

Temple, Dr. Allen D., Ste Anne's Hospital, Ste Anne de Bellevue, P.Q

Temple, Wallace George du, 77 N. Hill St., Port Arthur, Ont.

Tennyson, Brian D., 47 Cliffside Drive, Toronto.

Terry, Miss Mary Madeline, 189 Cedarvale Ave., Apt. 306. Toronto 13.

Thatcher, Max B., Dept. of Political Science, Univ, of Connecticut, Storrs, Conn.

Thériault, Noman, 160 Laurier, Laflèche, P.Q.

Thibault, Claude, Univ. of Sherbrooke, Sherbrooke, P.Q.

Thibault, René, 2515 Maplewood, App. 7, Montréal 26.

Thomas, L. G., Univ. of Alberta, Edmonton, Alberta.

Thomas, Lewis H., 9123 118th St., Edmonton, Alberta.

Thompson, D. Frederick, 2 Oakdale Drive N., Charleswood 20, Manitob'a.

Thompson, Fred F., Dept. of History, Royal" Military College, Kingston, Ont.

Thompson, Miss M. J., 415 59 Wilmot Place, Winnipeg 13, Manitoba.

Thomson, Prof. Dale C., Faculté des Sciences Sociales. Univ. de Montréal, Montréal 1 .

Thornton, A. P., Prof. of History, Univ. of Toronto. Toronto.

Thorpe, F. J., Chief Historian, National Museum of Canada, Ottawa.

Thorpe, William W., 11 Birch Court, Waggoners' Lane, Fredericton, N.B.

Thwaites, J. S., Residence Fellow - Fussell House, 1231 Colonel By Drive, Ottawa 1 .

Toews, Andrew, Box 718, Terrace, B.C.

Tolmie, Murray M., Dept. of History, Mount Allison Univ. Sackville, N.B.

Tomkins, George S., 3803 West 39th Avenue, Vancouver 13 .
Totton, S. J., 25 Hollinger Road, Toronto 16.

Towle, Prof. Edward $L$. Dept. of History, Univ. of Wisconsin-Milwaukee, Milwaukee, Wisconsin 53211.

Town, Henry H., 174 Lloy. minn Avenue, Ancaster,

Traviss, Stephen, 6 Ancroft Place, Toronto 5

Tremblay, Marcellin, 582 Lahaie, Pont Viau, Montréal 40.

Trudeau, Raoul, Bibliothécaire, Salaberry de Valleyfield, P.Q.

Trudel, Claude, 6642 - 41e avenue Ros., Montréal 36. Trudel, Marcel, Carleton Univ. Ottawa.

Trudel, Pierre, Département d'histoire, Univ. d'Ottawa, Ottawa.

Trueman, John, History Dept., McMaster Univ. Hamilton, Ont.

Tucker, Prof. Albert V., Dept. of History, Univ.' College, Univ. of Western Ontario, London, Ont.

Tulchinsky, G., Dept. of History, Univ. of Saskatchewan, Saskatoon.

Tumer, Allan R., Archives Division, Legislative Library, Regina, Saskatchewan.

Turner, Arthur C., Univ. of California, Riverside, California.

Turner, Harry E., McMaster Univ., Hamilton, Ont.

Turner, W. B., c/o Mrs. M. Tnmer, 2675 Bloor St. W. Toronto.

Tumer, William R., 37 Valley Road, Corner Brook, Newfoundland

Turp, Frederick J., 111 The West Mall, Apt. 303, Etobicoke, Ont.

Ullman, Walter, Dept. of History, Syracuse Univ., Syracuse, N.Y.

Unsinger, Peter C., 1024 South Blaine, Moscow Idaho 83843.

Upton, L. F. S., Dept. of pistory. F. S., Dept. of Columbia, Vancouver 8.

Usher, Mrs. Jean, 1873 Balsam, Apt. 2, Vancouver 9

Vanasse, Alfred R., Faculty of Arts, Univ. of Ottawa, Ottawa.

Vant, Miss Margaret, St. John's High School, Machray \& Salter St., Winnipeg.

Varosi, Rev. Ernest A. CR St. Jerome's High School,
100 Duke St. W., Kitchener, Ont.

Vennat, Pierre J. G.. 162 Willowdale Avenue, Outremont 8.

Vernon, Hugh, 24 Dumbar Road, Toronto 5.

Vervoort. Brother Donatus, P.O. Box 910, Taber, Alberta.
Vickey, R. B., 1805 - 108th Avenue, Dawson Creek,

Vogel, R., 4145 Marlowe, Montreal.

Voisine, Nive, prêtre Séminaire, Rimouski, P.Q

Vuckovic, M., Dept. of History, Univ. of Windsor,

Waite, Peter B., Dept. of $\mathrm{His}$ tory, Dalhousie Univ., Halifax, N.S

Waldon, Miss Freda, 36 Robinson St., Hamilton Ont.

Wales, Miss Mary A.' 31200 Stafford Drive, Birmingham, Michigan.

Walker, Dr. Frank N., 218 Inglewood Drive, Toronto 7

Walker, Lewie D., 5 Elm Avenue, Apt. 402, Toronto

Wallace, Carl M., Dept. of History, Laurentian Univ. Sudbury, Ont.

Wallot, Jean-Pierre, $385 \mathrm{St}$ Francis, Chateauguay, P.Q

Walmsley, N. E., Dept. of Political Science, Brandon College, Brandon, Man.

Walter, Miss Linda J.

Walter, T. D., 2442 Gladstone Ave. Windsor, Ont.

Ward, Rev. Érwin, 160' Stanley Ave., Ottawa.

Ward, W. P., 6111 - 111 Ave nue, Edmonton, Alberta

Warkentin, B. Álfred, 321A Westminster $\mathbf{N}$., Montreal. Warkentin, John H., Dept. of Geography, York' U., 2275 Bayview Ave. Toronto 12. Warner, Mrs. D. J. H., 30 Regent St., Lindsay, Ont.

Watson, Cicely, 154 Fenside Drive, Don Mills, Ont.

Watt, James $T_{\text {., }} 28$ Walter St., Toronto 13.

Waugh David, 38 Aldercrest Road, Dollard Des Ormeaux, P.Q

Webster, T. $\mathbf{S}$., Dept. of His tory, Univ. of Manitoba Winnipeg, Manitoba

Wedd, Stanley M., 144 Forest Hill Road, Toronto 7.

Weilbrenner, Bernard, 2118 Chemin Ste-Foy, Québec.

Weir, S. E., 486 Richmond St., London, Ont.

Whitby, Lieut. Commander J. P., R.C.N., 173 Oakridge Blvd., Ottawa 5.

White, Gerald Brian, 113 Madison Ave Toronto 5 White, Harry, 1 Woodcrest Drive, R.R. 1, Islington, Ont.

White, P. C. T., Dept. of History, Univ. of Toronto, Toronto 5.

White, Walter S., 67 Cheval du Moine, Ste Anne de Sorel, P.Q.

Whitelaw, $\dot{W}$. M., 4 Ridge Road, Carmel, N.Y.

Whiteley, W. H. Apt. 221, 4675 Imperial St., Burnaby 1, B.C.

Whiteside, Miss M. S., 15

Lapthorne Ave., Charlotte.

town, P.E.I. 
Whittard, Miss Bessie N, 19 Syke Ings, Iver, Bucks, England.

Wiedner, Dr. Donald L. Dept. of History, Univ. of Alberta, Edmontón, Alberta

Wilbur, J. Richard H. 750 McEvoy St., Fredericton, N.B.

Wiley, Gerald E., 356 Clarendon Drive, Ancaster, Ont.

Wilkinson, Prof. B., Dept. of History, Univ. of Toronto, Toronto 5.

Williams, C. Brian, Faculty of Commerce, Univ. of Alberta, Edmonton, Alberta. Werta, Ednief Justice E. K.

291 Yale Ave., Winnipeg. Manitoba.

Williams, John A., Dept. of History, Univ. of Washing ton, Seattle, Washington 98115 .

Williams, R. J., Copp Clark Publishing lington St. West, Toronto lingt

Willis, N. M., Dept. of History, Sidney Smith Hall Univ. of Toronto, Toronto 2.

Willoughby, William R., 85 Harewood Crescent, Fredericton, N.B.

wills, Harold A., c/o Northland Post, Box 110, Coland Post,

chrane, Ont. Dept. of History, Trent Univ., Peterborough, Ont.

Wilson Miss Barbara $M$. Public Archives of Canada, Ottawa.
Wilson Donald J. 74 West St. N., Thorold, Ont

Wilson, J. Donald, College of Education, Univ. of Western Ontario, London, Ont.

Wilson, J. M., c/o Dept. of Political Science, Univ. of Waterloo, Waterloo, Ont.

Wilson Richard D 5805 Darlington Ave., Montreal. Winch, Mrs. Mary E., c/o D. M. Winch, Dept. of Political Economy, Univ. of Toronto, Toronto 5.

Windsor, K. N., Massey College, Toronto 5.

Winks, R. W., Dept. of Hiscory, Yale Univ., New Haven, Conn.

Winter, Dr. Carl G., 126 43rd' St., Sacramento 19, California.

Wirick, Prof. Arthur J., Extension Dept., Univ. of Saskatchewan, Saskatoon.

Wise, S. F Dept. of History, Queen's Univ., Kingston, Ont.

Wishart, Rev. Ian, 1900 MacPherson Ave., Regina, Saskatchewan.

Wohler, J P 1324 Brown Blvd., Verdun, Montreal 19. Wolff, Carl M., Asst. Prof., Brock Univ., St. Catharines, Ont.

Wolf, Mrs. Helen, 9379 LaSalle Blvd., Apt. 206, LaSalle, P.Q.

Wrenn, Miss Phyllis M., 88 Homewood Avenue, Apt. 101, Toronto 5.
Wright, Mrs. Esther, Public Archives of Canada, Ottawa 2.

Wright, Peter, R.R. 1, King, Ont.

Wynne, Robert E., Dept. of History, Univ. of Waterloo, Waterloo, Ont.

Yandle, Mrs. Anne, Special Collections Division, Univ. of British Columbia, Vancouver 8.

Young, D. M., Dept. of History, Univ. of New Brunswick, Fredericton, N.B.

Young, J. C., Executive Editor, Encyclopedia Canadiana, 1300 Carling Ave. Ottawa 3.

Young, Prof. W. D., Dept. of Economics \& Political Science, Univ. of British Columbia, Vancouver 8 .

Younge, Miss Eva, 8680 Montcalm St., Suite 4, Vancouver 14, B.C.

Younker, D. W., Dept of History, St. Cloud State College, St. Cloud, Minnesota.

Zacour, Dr. Norman $P$. Dept, of History, Franklin and Marshall College, Lancaster, $\mathbf{P a}$.

Zaslow, Prof. Morris, Dept. of History, Middlesex College, Univ. of Western Ontario, London, Ont.

Zia, Abdul Q., Dept. of History, Laurentian Univ. Sudbury, Ont.

Zoltyany, Yves F., Dept. of History, Univ. of Waterloo, Waterloo, Ont. 